\title{
O USO DA ULTRA-SONOGRAFIA NO DIAGNÓSTICO E EVOLUÇÃO DA APENDICITE AGUDA*
}

\author{
Arquimedes Artur Zorzetto ${ }^{1}$, Linei Augusta Brolini Dellê Urban², Christian Bark Liu², Olívia \\ Russo Cruz ${ }^{3}$, Maria Luiza Malfi Vitola ${ }^{1}$, Yumi Awamura ${ }^{4}$, Alessandra Bettega Nascimento ${ }^{4}$
}

Resumo OBJETIVO: Aproximadamente 35\% das apendicites agudas têm diagnóstico clínico pré-operatório duvidoso ou incorreto, particularmente grávidas e crianças. A ultra-sonografia, em virtude do seu baixo custo e facilidade de acesso, tem-se mostrado um método diagnóstico importante. Este estudo propôs-se a demonstrar os principais achados de imagem das diversas fases da apendicite, com o objetivo de auxiliar o ultra-sonografista no diagnóstico precoce desta afecção. MATERIAIS E MÉTODOS: São relatados 14 casos de ultrasonografias abdominais realizadas no período de janeiro a julho de 2001, em pacientes que se apresentavam com quadro de abdome agudo. $\mathrm{O}$ exame foi realizado com transdutores de 3,5 $\mathrm{MHz}$ e 7,5 $\mathrm{MHz}$. RESULTADOS: $O$ estudo ultra-sonográfico antes da perfuração demonstra apêndice não compressível, com espessamento e perda focal da definição das paredes. Após a perfuração, o apêndice pode não ser visualizado ao exame de ultra-sonografia, sendo evidenciadas alterações secundárias como efeito de massa, formação de plastrão, liquefação e formação de abscesso, além de ar dentro da coleção. CONCLUSÃO: 0 diagnóstico precoce da apendicite é essencial para minimizar a morbidade, que se mantém elevada se ocorrer perfuração. Apresentações atípicas resultam em confusão diagnóstica e retarde no tratamento. As principais dificuldades e erros são apendicite retrocecal, apendicite focal ou perfurada.

Unitermos: Apendicite; Ultra-sonografia; Tomografia computadorizada.

Abstract Ultrasonography in the diagnosis and evolution of acute appendicitis.

OBJECTIVE: Approximately $35 \%$ of the patients with acute appendicitis have a doubtful or incorrect preoperatory diagnosis, particularly in pregnant women and children. Ultrasonography has demonstrated to be an important means of diagnosis due to its low cost and easy access. This study is intended to show the main imaging findings of the many phases of appendicitis, thus helping the ultrasonographist to establish an early diagnosis. MATERIALS AND METHODS: From January to June 2001, 14 patients with acute abdominal pain were submitted to abdominal ultrasound using $3.5 \mathrm{MHz}$ and $7.5 \mathrm{MHz}$ transducers. RESULTS: The ultrasound findings when perforation had not occurred demonstrated a noncompressible appendix, thickening and focal lack of definition of the walls. After perforation had occurred the appendix could no longer be visualized at ultrasonography. Secondary changes such as mass effect, phlegmous changes, liquefaction and abscess formation, and gas bubbles within a collection were identified. CONCLUSION: An early diagnosis of appendicitis is essential in order to minimize morbidity that is high when perforation occurs. Atypical presentations result in misdiagnosis and treatment delay. The main diagnostic challenges are retrocecal, focal or perforated appendicitis.

Key words: Appendicitis; Ultrasonography; Computed tomography.

\section{INTRODUÇÃO}

A apendicite aguda é a causa mais comum de abdome agudo. Embora muitos

* Trabalho realizado no Serviço de Radiologia do Hospital de Clínicas da Universidade Federal do Paraná (UFPR) e na CedipClínica de Diagnóstico por Imagem do Paraná, Curitiba, PR.

1. Médicos do Serviço de Radiologia do Hospital de Clínicas da UFPR e da Cedip - Clínica de Diagnóstico por Imagem do Paraná.

2. Médicos Residentes do Serviço de Radiologia do Hospital de Clínicas da UFPR.

3. Acadêmica de Medicina do Hospital de Clínicas da UFPR. 4. Médicos da Cedip - Clínica de Diagnóstico por Imagem do Paraná.

Endereço para correspondência: Dr. Arquimedes A. Zorzetto. Cedip. Rua Rafael Papa, 20, Jardim Social. Curitiba, PR. E-mail: aazorzetto@yahoo.com.br

Recebido para publicação em 10/1/2002. Aceito, após revisão, em 11/11/2002. pacientes apresentem quadro inicial clássico, alguns têm manifestações atípicas que dificultam o diagnóstico, particularmente gestantes e crianças $^{(\mathbf{1})}$. Em aproximadamente $35 \%$ dos casos a apendicite já está em fase adiantada, com perfuração e abscesso local, no momento da cirurgia ${ }^{(2)}$. Igualmente inquietante é o fato de que $16 \%$ a $47 \%$ dos casos das laparotomias de emergência de um hospital geral são brancas, ou seja, acarretam a remoção de apêndices normais $^{(3)}$.

Vários estudos foram feitos para aperfeiçoar os critérios e estabelecer o valor da ultra-sonografia na avaliação de pacientes com evidências duvidosas dessa doença, levando a uma diminuição do número de laparotomias negativas ${ }^{(\mathbf{4 , 5})}$.

Este estudo propôs-se a demonstrar os principais achados de imagem das diversas fases da apendicite, com o objetivo de auxiliar o ultra-sonografista no diagnóstico precoce desta afecção.

\section{MATERIAIS E MÉTODOS}

São relatados 14 casos de ultra-sonografias abdominais realizadas no período de janeiro a julho de 2001, em pacientes provenientes do Pronto Atendimento do Hospital de Clínicas da Universidade Federal do Paraná, que se apresentavam com 
quadro de dor abdominal difusa ou localizada na fossa ilíaca direita, além de hemograma infeccioso e radiografias simples com achados inespecíficos. $\mathrm{O}$ exame foi realizado inicialmente com transdutores de 3,5 $\mathrm{MHz}$ e complementado com transdutores de 7,5 $\mathrm{MHz}$.

\section{RESULTADOS}

O estudo ultra-sonográfico demonstrou, nas fases iniciais, apêndice com diâmetro aumentado (76\%) (Figura 1), não compressível (73\%) (Figura 2), paredes espessadas (53\%) (Figura 3), além de per- da focal da definição das paredes (23\%) (Figura 4) e pequena quantidade de líquido ao redor (47\%) (Figuras 5 e 6). Em casos mais avançados, observaram-se formação de plastrão (15\%) (Figura 7), liquefação (7\%) (Figura 8) e formação de abscesso $(5 \%)$ (Figura 9), além de ar dentro da

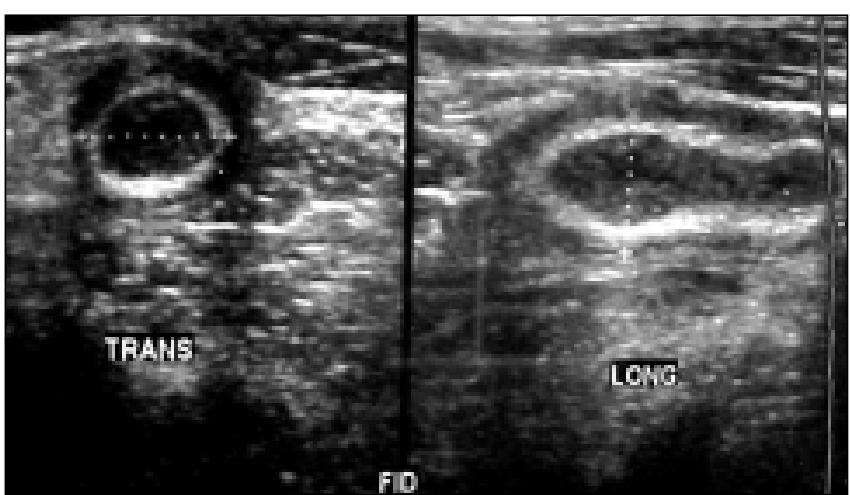

A

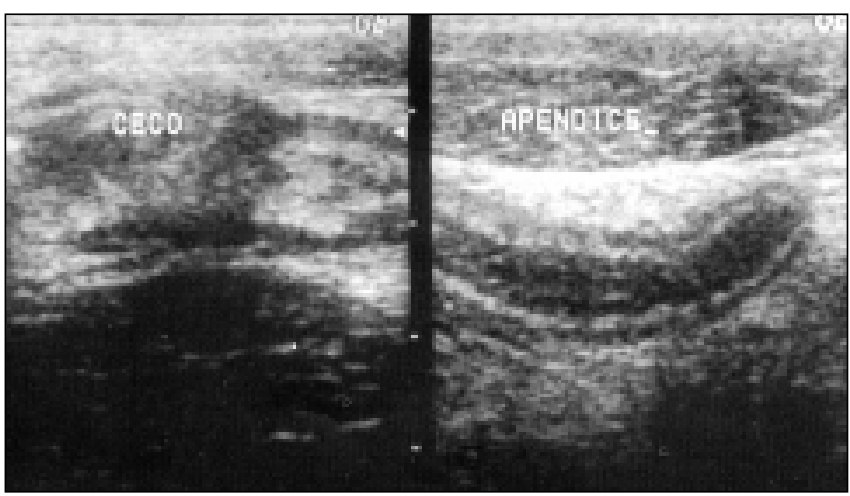

Figura 2. Paciente do sexo masculino, 32 anos de idade. Apêndice medindo $8 \mathrm{~mm}$, sem mudança na conformação após compressão localizada.

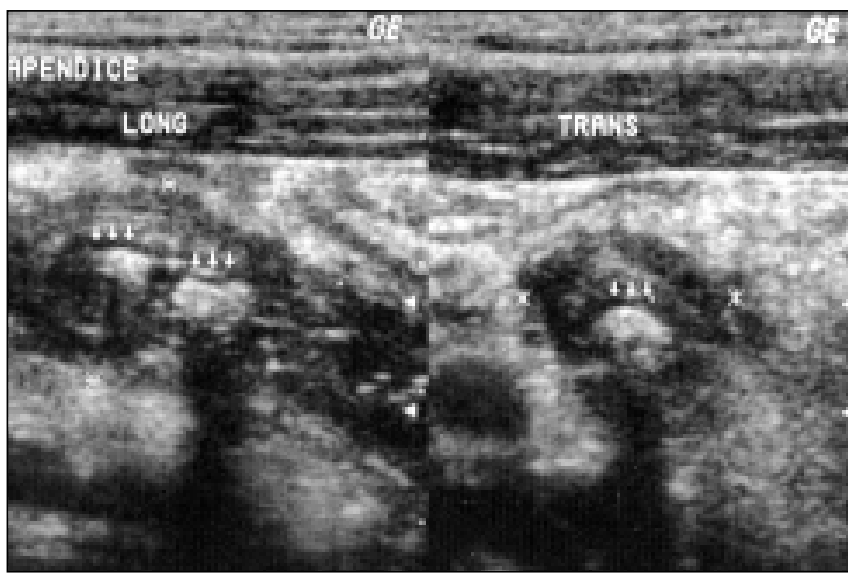

Figura 4. Paciente do sexo masculino, 16 anos de idade. Apêndice com imagem ecogênica com sombra acústica no interior (apendicolito), além de líquido ao redor.

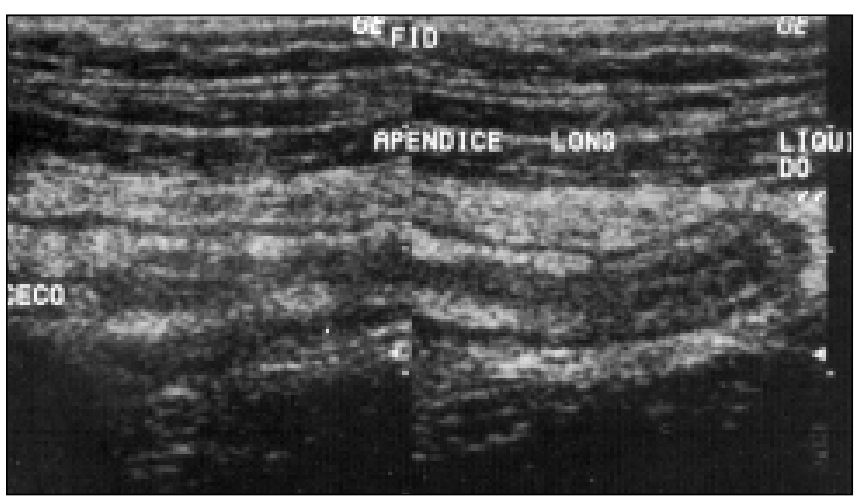

B

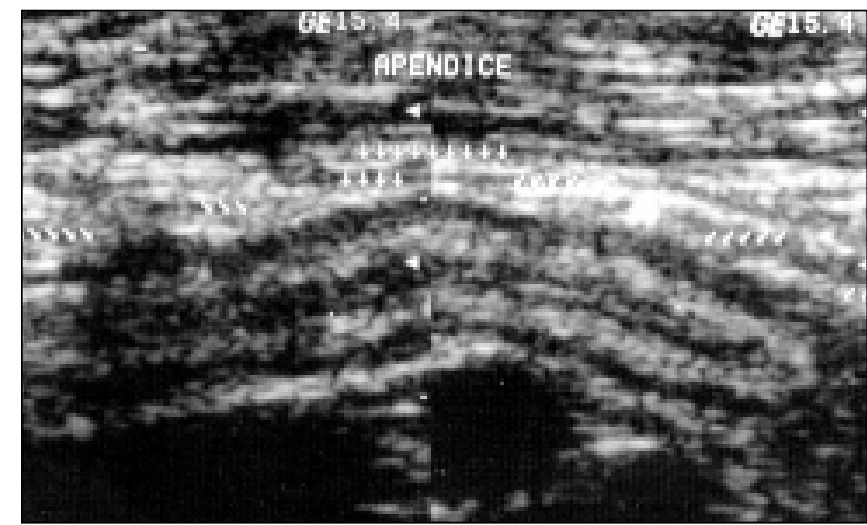

Figura 3. Paciente do sexo masculino, 38 anos de idade. Apêndice com paredes espessadas $(4 \mathrm{~mm}$ ) e perda da estratificação habitual.

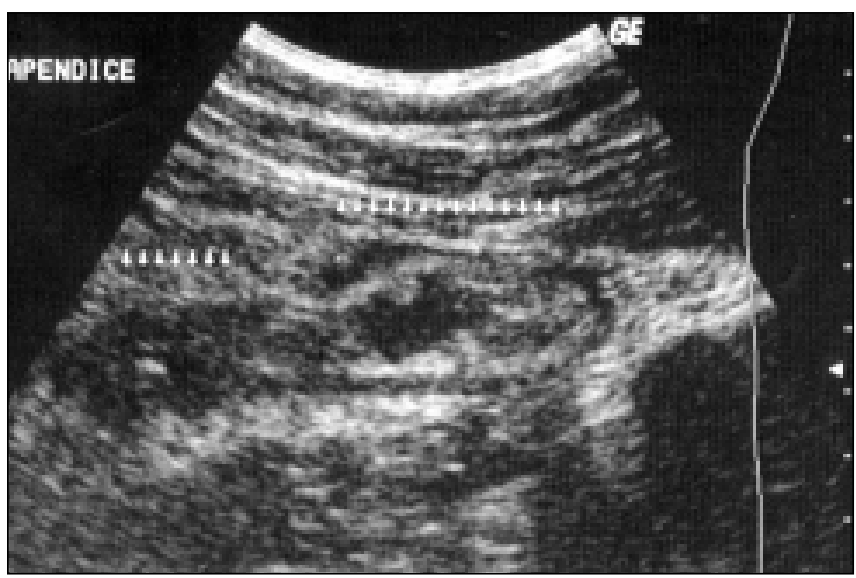

Figura 5. Paciente do sexo feminino, 24 anos de idade. Imagem tubuliforme, com paredes espessadas e perda da definição na porção proximal, sugestivo de perfuração. 


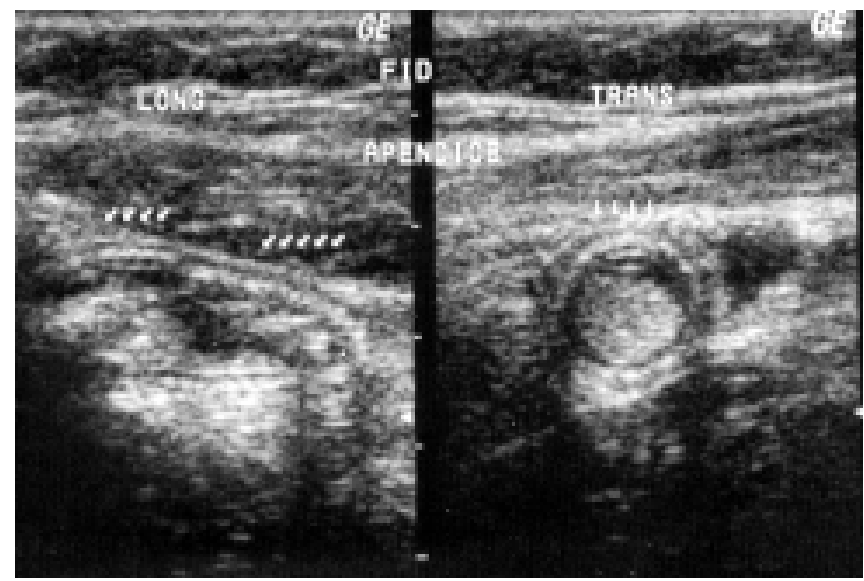

Figura 6. Paciente do sexo masculino, 32 anos de idade. Apêndice de calibre aumentado (13 mm), com conteúdo dismórfico no interior e pequena coleção líquida adjacente.

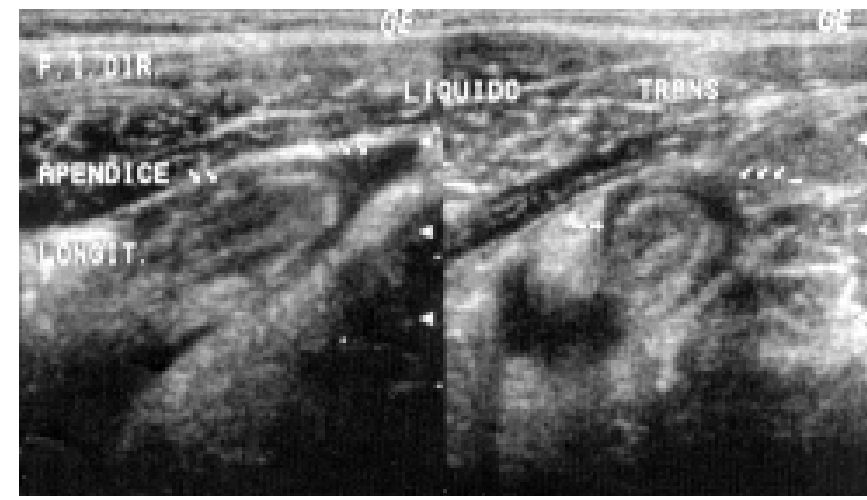

Figura 7. Paciente do sexo masculino, 23 anos de idade. Observa-se pequena quantidade de líquido ao redor do apêndice.
Figura 8. Paciente do sexo masculino, 44 anos de idade. Imagem hipoecóica e mal delimitada na fossa ilíaca direita, medindo aproximadamente $3 \mathrm{~cm}$ no maior diâmetro.

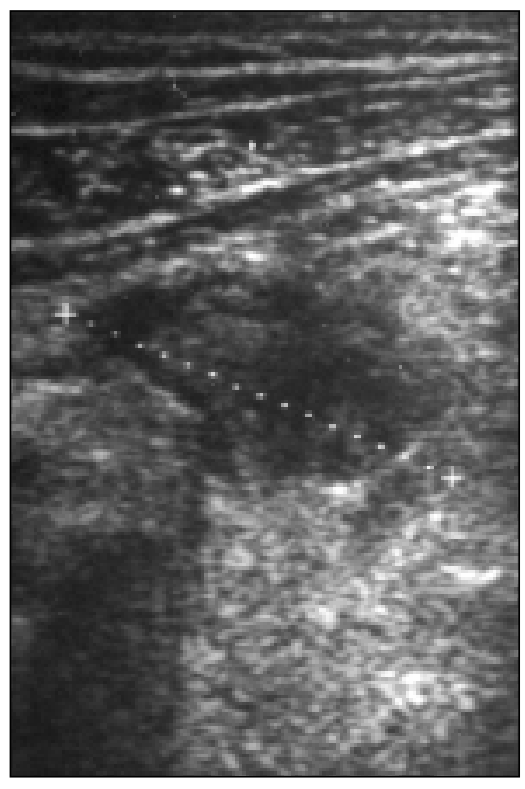

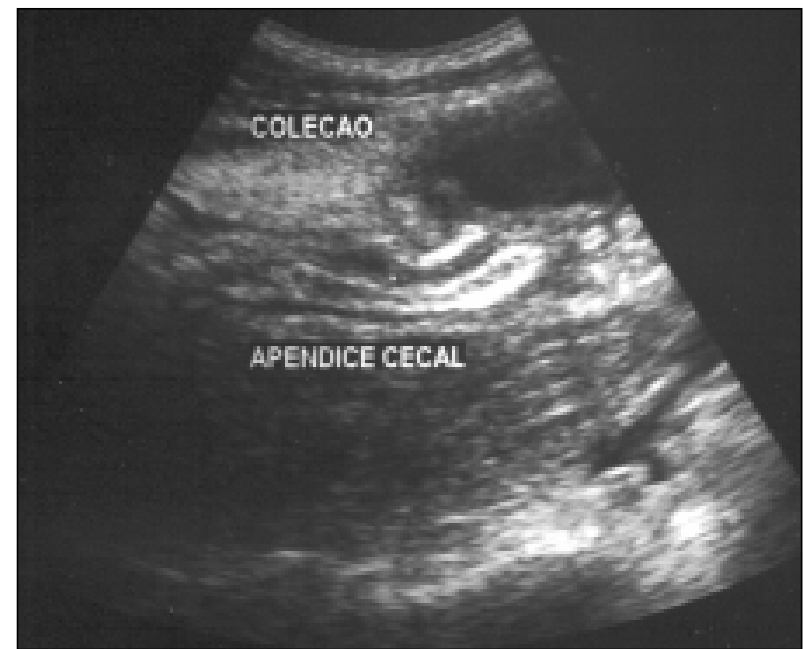

Figura 9. Paciente do sexo masculino, 27 anos de idade. Observa-se coleção hipoecóica, mal delimitada na fossa ilíaca direita, adjacente à porção terminal do apêndice. coleção (1\%) (Figura 10). Observou-se, ainda, apêndice complicado em uma gestante de 23 semanas (Figura 11), em uma criança com um ano e meio de idade (Figura 12) e em um caso na região retrocecal (Figura 13).

\section{DISCUSSÃO}

O apêndice normal raramente é visto, sendo observado principalmente nos casos de ascite ou conteúdo fecal espesso. Pode ser visualizado como um órgão tubular, com diâmetro menor que $6 \mathrm{~mm}$ e paredes estratificadas menores que $3 \mathrm{~mm}^{(\mathbf{1})}$.

A fisiopatologia da apendicite aguda inicia com a obstrução do lúmen apendi- cular, por material fecal ou apendicolito

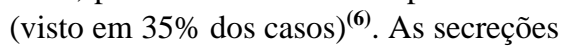
mucosas aumentam a pressão intraluminal e comprometem o retorno venoso. A mucosa torna-se hipóxica e apresenta ulcerações. Sobrevém infecção bacteriana, culminando, por fim, em gangrena e perfuração. Ocorre então peritonite livre, podendo ser vistas coleções na pelve, flanco, regiões sub-hepáticas e subdiafragmáticas à direita, porém mais comumente observa-se abscesso encapsulado ao redor do apêndice, devido a bloqueio do processo pelo mesentério e alças da região ${ }^{(7)}$.

O diagnóstico ultra-sonográfico da apendicite aguda depende do estágio anatomopatológico desta. $\mathrm{Na}$ apendicite aguda

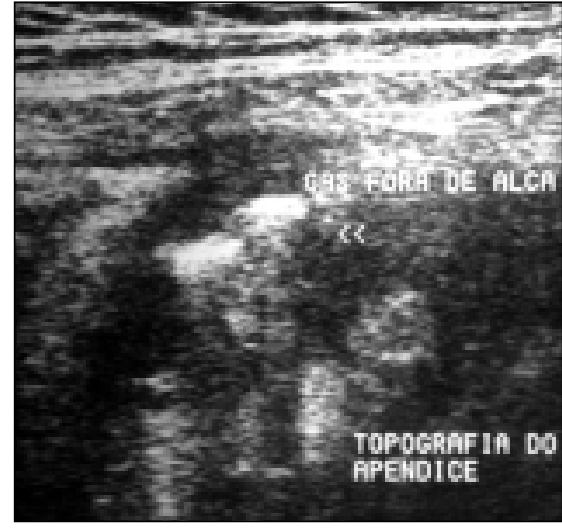

Figura 10. Paciente do sexo feminino, 36 anos de idade, com exame ultra-sonográfico mostrando, na fossa ilíaca direita, coleção hipoecóica, mal delimitada, com áreas ecogênicas no interior, compatível com ar fora de alça intestinal. 


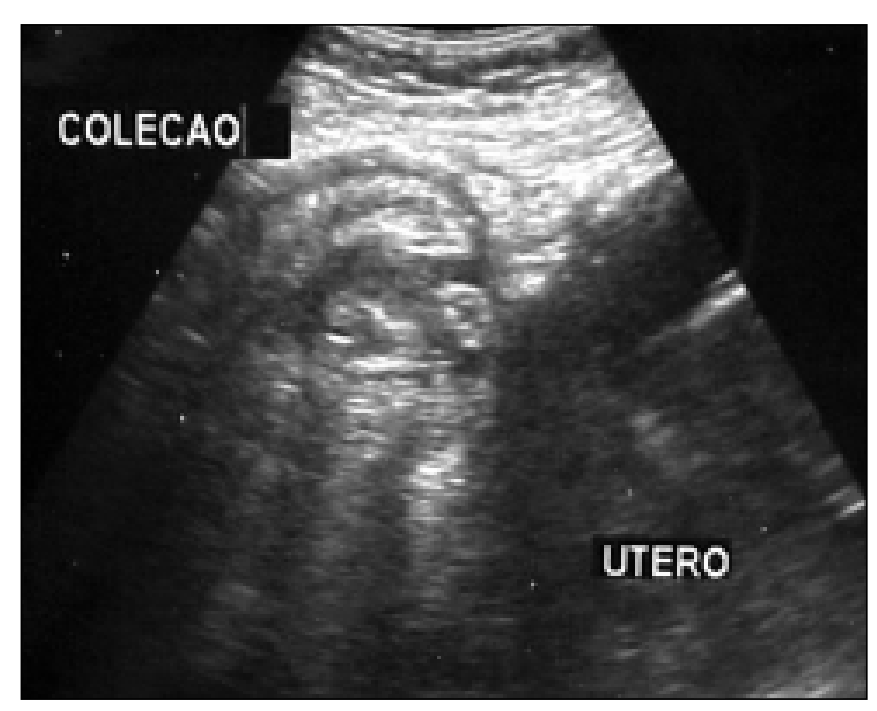

Figura 11. Gestante de 18 semanas, com 19 anos de idade, apresentando, há uma semana, dor de leve intensidade na fossa ilíaca direita. Observava-se, adjacente ao útero, coleção hipoecóica, mal delimitada, com ar no interior, medindo aproximadamente $4 \mathrm{~cm}$ no maior diâmetro.

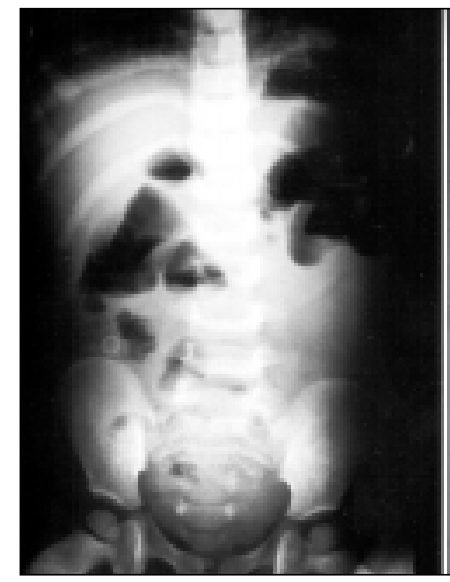

A

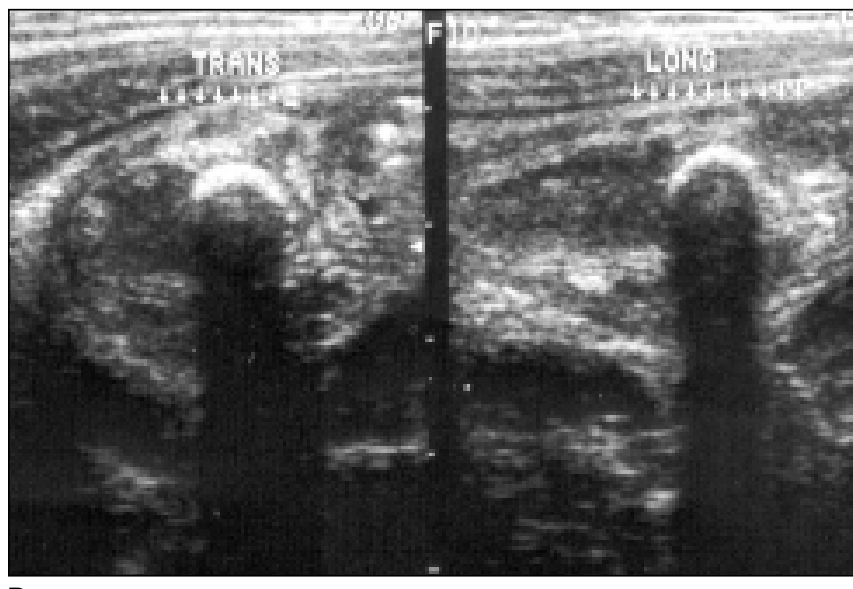

B

Figura 12. Criança com um ano de idade, apresentando dor adominal há 12 horas. A radiografia simples demonstra imagem densa e arredondadana fossa ilíaca direita, compatível com apendicolito (A). Foi complementado o estudo com ultra-sonografia, que demonstrou imagem tubuliforme, com apendicolito no interior e líquido ao redor, compatível com apendicite perfurada (B).

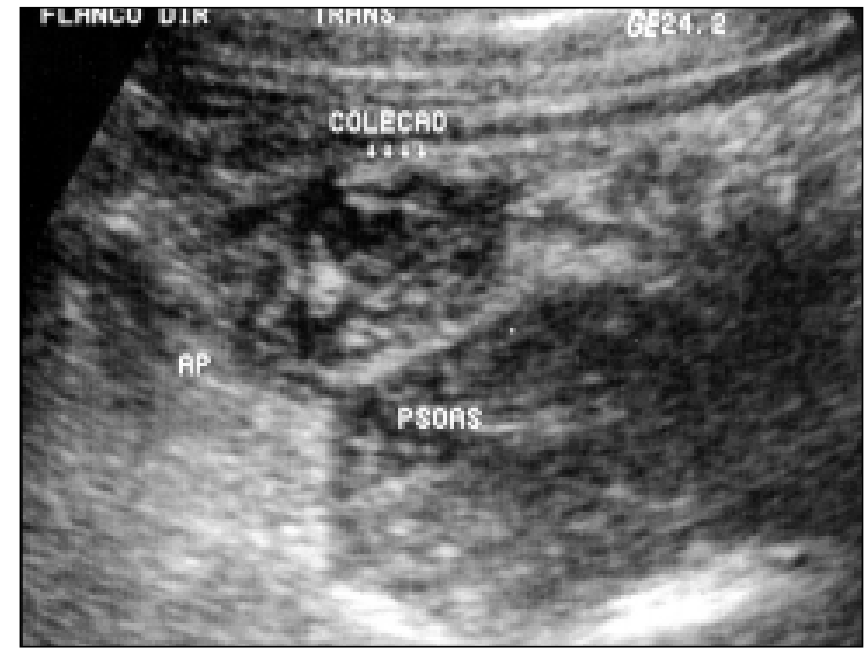

A

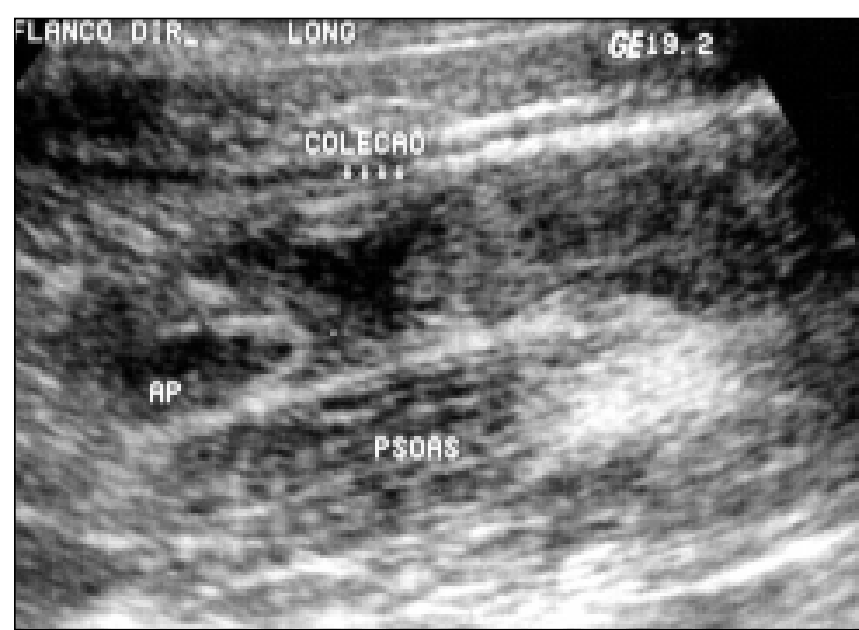

B

Figura 13. Paciente do sexo masculino, 24 anos de idade. Observa-se coleção hipoecóica mal delimitada localizada sobre o músculo iliopsoas à direita (A), compatível com apêndice retrocecal (B). 
simples o órgão conserva sua forma, porém apresenta-se edemaciado em toda a sua extensão. Os principais achados ultra-sonográficos são: apêndice não compressível com diâmetro transversal maior que $6 \mathrm{~mm}$, paredes com espessura maior que $3 \mathrm{~mm}$, ausência da camada ecogênica central (submucosa), ausência de gás no interior do apêndice, sendo este preenchido por líquido, presença de apendicolito, visível como uma imagem ecogênica com sombra acústica, independentemente do tamanho apendicular ${ }^{(7)}$.

$\mathrm{Na}$ fase da apendicite gangrenosa observa-se necrose localizada ou extensa da parede apendicular, às vezes com perfurações microscópicas. No exame por ultra-som podem ser observados espessamento irregular da parede do apêndice, pequenas coleções intraparietais, anecóicas ou ecogênicas, causadas por pequenos abscessos ou ar, ou ainda pequenas coleções periapendiculares ${ }^{(8)}$.

$\mathrm{Na}$ fase de apendicite francamente perfurada ocorre rompimento da parede com extravasamento do conteúdo para a cavidade abdominal, muitas vezes com destruição total do apêndice. Nesta fase, a sensibilidade do ultra-som diminui, porque não se visualiza mais o apêndice, mas apenas as alterações adjacentes, como massa pe- riapendicular, coleções intra-abdominais ou adenopatia mesentérica. Os abscessos, forma mais comum de apresentação da perfuração, podem apresentar-se de formas variadas, desde imagens anecóicas semelhantes a cistos, até imagens complexas, mal definidas, acompanhadas de sombra acústica causada pelo ar em cavidade. Podem simular alças intestinais, com diferenciação através da observação dos "movimentos brownianos", característicos de alças intestinais ${ }^{(\mathbf{9})}$.

As principais dificuldades e fontes de erros no diagnóstico da apendicite são: exames de pobre qualidade técnica, com transdutores de baixa frequiência ou com profissionais não habilitados; exames incompletos, que podem levar à falsa impressão de apêndice normal quando, na verdade, a inflamação está confinada à sua ponta; a posição do apêndice também é causa de erros, particularmente quando é retrocecal; pacientes obesos e com distensão abdominal acentuada também podem levar a diagnóstico falso-negativo ${ }^{(\mathbf{1 0})}$.

Trabalhos recentes, visando ao diagnóstico de apendicite por ultra-sonografia, indicam sensibilidade do método entre $68 \%$ e $89 \%$, especificidade entre $95 \%$ e $100 \%$, com valor preditivo positivo entre $89 \%$ e $96 \%$ e valor preditivo negativo entre $76 \%$ e $96 \%$, demonstrando ser a ultra-sonografia um excelente método diagnóstico nos casos de apendicite aguda e de suas complicações $^{(11)}$.

\section{REFERÊNCIAS}

1. Puylaert JBCM. Acute appendicitis: US evaluation using graded compression. Radiology 1986;158: 355-60.

2. Shaw RE. Appendix calculi and acute appendicitis. Br J Surg 1965;52:452-9.

3. Jeffrey RB Jr, Laing FC, Lewis FR. Acute appendicitis: high-resolution real- time US findings. Radiology 1987;163:11-4.

4. Dachman AH, Nichols JB, Patrick DH, Lichtenstein JE. Natural history of the obstructed rabbit appendix: observations with radiography, sonography, and computed tomography. AJR 1987;148:281-4.

5. Jeffrey RB Jr, Laing FC, Townsend RR. Acute appendicitis: sonographic criteria based on 250 cases. Radiology 1988;167:327-9.

6. Abu-Yousef MM, Bleicher JJ, Maher JW, Urdaneta LF, Franken EA Jr, Metcalf AM. High-resolution sonography of acute appendicitis. AJR 1987;149: 53-8.

7. Rioux M. Sonographic detection of the normal and abnormal appendix. AJR 1992;158: 773-8.

8. Sheng PY. Apendicite aguda gangrenosa com abscesso. Rev Imagem 1986;8:61-2.

9. Borushok KF, Jeffrey RB Jr, Laing FC, Townsend RR. Sonographic diagnosis of perforation in patients with acute appendicitis. AJR 1990;154:2758.

10. Lim HK, Lee WJ, Lee SJ, Namgung S, Lim JH Focal appendicitis confined to the tip: diagnosis at US. Radiology 1996;200:799-801.

11. Birnbaum BA, Wilson SR. Appendicitis at the millenium. Radiology 2000;215:337-48. 